\title{
Wells-Syndrom (eosinophile Zellulitis)
}

\section{B. Khan Durani \\ J. Wacker \\ W. Hartschuh}

Wells Syndrome (Eosinophilic Cellulitis)

\section{Zusammenfassung}

Die eosinophile Zellulitis ist eine seltene Hauterkrankung unklarer Genese, welche klinisch durch rasches Auftreten von zum Teil stark juckenden, urtikariellen Plaques charakterisiert ist. Das Wells-Syndrom sollte bei urtikariellen Hautveränderungen als seltene Differenzialdiagnose neben der bakteriellen Zellulitis, Arthropodenreaktionen, Urtikaria, Urtikariavaskulitis und dem Hypereosinophilie-Syndrom berücksichtigt werden. Die Diagnose wird durch das klinische Erscheinungsbild und die Histologie mit typischen „flame figures“ gesichert. Andere Systemerkrankungen, die gehäuft mit dem Wells-Syndrom einhergehen, sollten ausgeschlossen werden. Therapeutisch sind zunächst lokale und/oder systemische Kortikosteroide zu empfehlen.

\section{Abstract}

Eosinophilic cellulitis is a rare skin disease of unknown aetiology, clinically resembling acute bacterial cellulitis, arthropod bites, urticaria, urticarial vasculitis and hypereosinophilic syndrome. The diagnosis is based on the clinical presentation and the histology of eosinophilic infiltration and "flame figures" in the dermis. Associated systemic diseases including leukaemia, rheumatoid arthritis and non-haematological malignancy should be excluded. Treatment of choice is local or low-dose oral corticosteroids.

\section{Kasuistik}

ton Wells beschrieben [1]. Er berichtete über 4 Patienten mit einer „rezidivierenden granulomatösen Dermatitis mit Eosinophilie“. 1979 wurde von Wells und Smith durch die Veröffentlichung von weiteren 8 Fällen der Name „eosinophile Zellulitis“ geprägt [2].

Die Hautveränderungen beginnen häufig als erythematöse, urtikarielle Ödeme mit z.T. massivem Juckreiz oder Brennen und können sich großflächig ausbreiten. Im Verlauf ist die Bildung von Blasen möglich. Selten treten Fieber und Gelenkschmerzen hinzu. Nach Tagen bis Wochen kommt es zu einer langsamen Abheilung mit Änderung der Farbe von hellrot über rotbraun zu blaugrün. Rezidive sind jedoch nicht selten.

\section{Anamnese}

Eine 39-jährige Patientin stellt sich mit einer seit 7 Tagen bestehenden, stark juckenden Hautveränderung an der rechten Flanke in unserer Ambulanz vor. Diese habe sich im Verlauf ausgedehnt und vereinzelt seien Blasen aufgetreten. Fieber oder Abgeschlagenheit bestehen nicht.

\section{Hautstatus}

An der rechten Flanke zeigt sich ein ausgedehntes, asymmetrisches, erythematöses, zentral teils livides, urtikarielles Infiltrat mit zumeist scharfer Abgrenzung zum Rand (Abb. 1 und 2). Vereinzelt sind bis zu $1 \times 1 \mathrm{~cm}$ große Blasen zu sehen. 


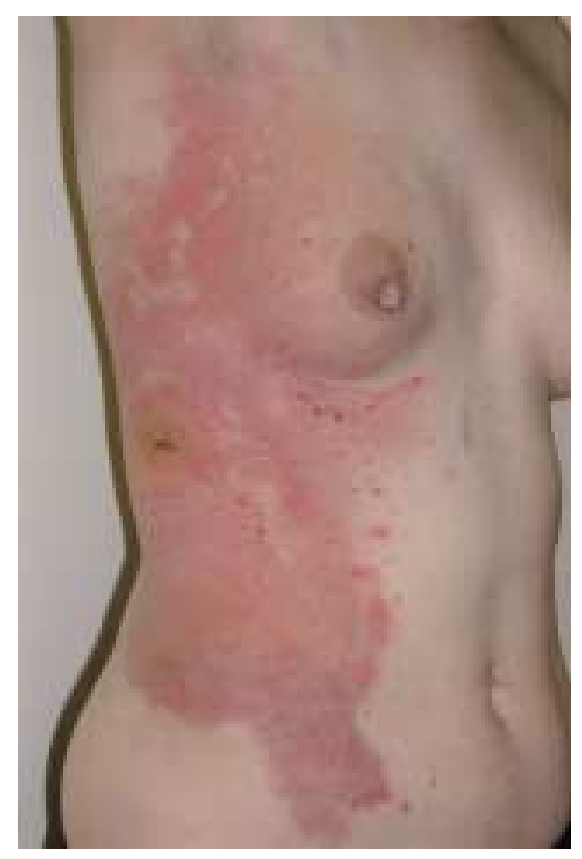

Abb. 1 Ausgeprägtes urtikarielles Infiltrat der rechten Flanke mit einzeln stehenden, prallen Blasen.

Abb. 2 Detailansicht der Blasen.

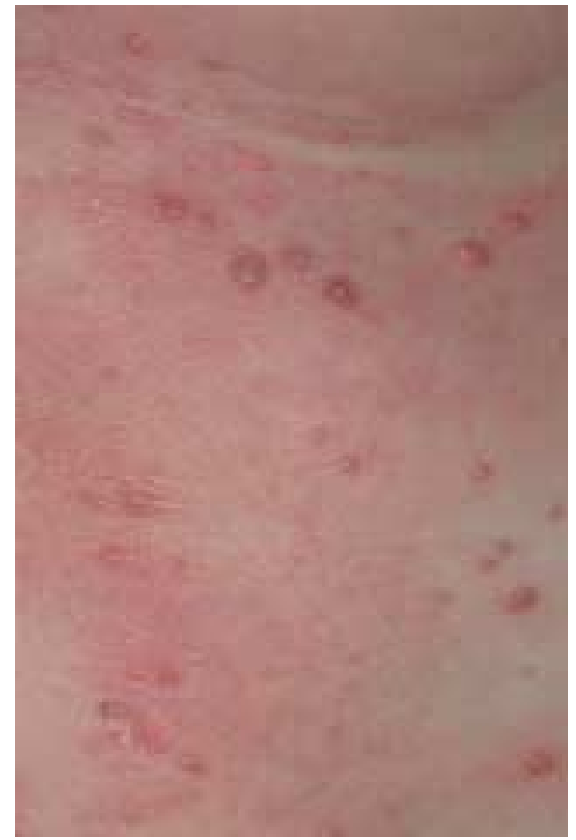

\section{Befunde}

Blutbild, Leberwerte, Nierenwerte, Ferritin, ANA, ENA, ANCA, Cardiolipin-Antikörper und Lupus-Antikoagulans liegen im Normbereich. Röntgenthorax und Oberbauchsonographie sind unauffällig.

\section{Histologie}

Unter einer regelrecht geschichteten Epidermis findet sich in den Papillenspitzen ein stark ausgeprägtes Ödem. In allen Coriumetagen, vorzugsweise jedoch im oberen Corium, zeigt sich ein kräftiges perivaskuläres und interstitielles Infiltrat aus massenhaft eosinophilen Granulozyten und Lymphozyten. Die eosinophilen Granulozyten umlagern teilweise kondensiert wirkende Kollagenfasern im Sinne von „flame figures“ (Abb. 3).

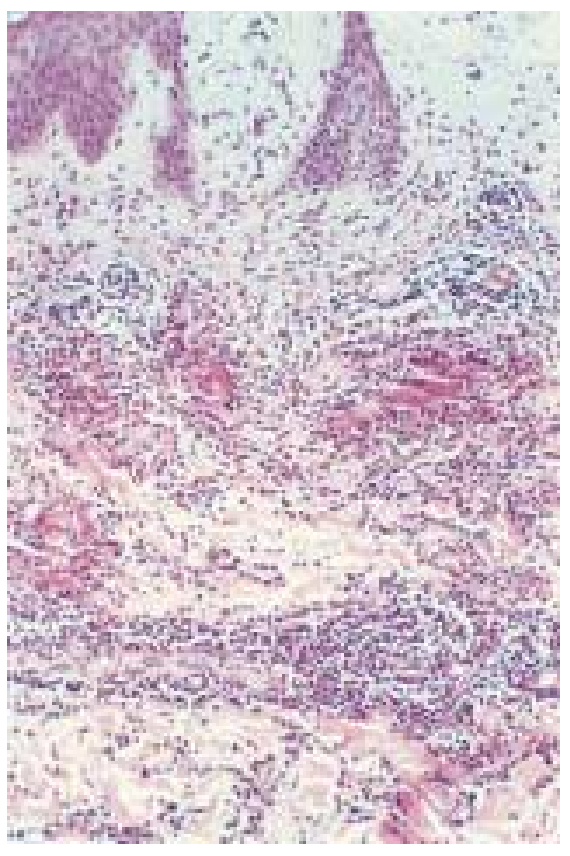

Abb. 3 In der Histologie zeigt sich ein ausgeprägtes Ödem der Papillenspitzen. Im Corium sieht man ein kräftiges, perivaskuläres und interstitielles Infiltrat aus massenhaft eosinophilen Granulozyten und Lymphozyten. Die eosinophilen Granulozyten umlagern teilweise kondensiert wirkende Kollagenfasern im Sinne von „flame figures“.

\section{Therapie und Verlauf}

Unter lokaler Therapie mit einer Betamethason-haltigen Zinkschüttelmixtur zeigte sich innerhalb weniger Tage eine Abblassung der Hautveränderungen. Eine vollständige Abheilung war nach einer Woche zu beobachten.

\section{Diskussion}

Die Diagnose einer bullösen Variante der „eosinophilen Zellulitis“ oder Wells-Syndrom wurde bei dieser Patientin aufgrund des klinischen Erscheinungsbildes mit urtikariellen, stark juckenden erythematösen Plaques mit teils hämorrhagischen Blasen und der histologisch nachweisbaren eosinophilenreichen Infiltrate und Flammenfiguren in der Dermis gestellt.

Das Wells-Syndrom ist eine Erkrankung unklarer Genese. Klinisch präsentieren sich erythematöse, urtikarielle, großflächig konfluierende, scharf begrenzte Ödeme mit z.T. massivem Juckreiz. Die Hautveränderungen entstehen innerhalb von 2 - 3 Tagen und heilen häufig spontan innerhalb von 2-8 Wochen ohne Narbenbildung ab [2]. Blasen können auftreten. Über einen Verlauf in den Blaschko-Linien wurde berichtet [3]. Seltener werden einzeln stehende, rötliche Knoten als einzige Manifestation beschrieben [4].

Aufgrund der häufigen Assoziation von urtikariellen Reaktionen, Eosinophilie und möglichen Triggerfaktoren durch Insekten oder Parasiten wurde das Wells-Syndrom auch als Ausdruck einer allergischen Überempfindlichkeitsreaktion auf verschiedene Stimuli angesehen [5-7].

Die klinische Differenzialdiagnose umfasst die bakterielle Zellulitis, Arthropodenreaktionen, Urtikaria, Urtikariavaskulitis und das Hypereosinophilie-Syndrom. 
Histologisch findet man in akuten Läsionen ein eosinophilenreiches, dermales Infiltrat. Die Blasenbildung entsteht durch ein dermales Ödem. Eine Degranulation von Eosinophilen führen zu Denaturierung von Kollagenfasern, die als „Flammenfiguren“ bezeichnet werden [8]. Die Flammenfiguren stellen hierbei ein histologisches Reaktionsmuster der Haut bei eosinophilenreichen Dermatosen dar, das jedoch nicht spezifisch für das Wells-Syndrom ist. Sie können auch bei Prurigo nodularis, Ekzemen, dem bullösen Pemphigoid, Herpes gestationis oder Infektionen durch Dermatophyten beobachtet werden.

Im späteren Verlauf dominieren Histiozyten und Riesenzellen das histologische Bild. Eine Vaskulitis wird nicht gefunden.

Die Assoziation des Wells-Syndroms mit anderen eosinophilenreichen Erkrankungen wie dem Hypereosinophilie-Syndrom und der Churg-Strauss-Vaskulitis wurde beschrieben $[9,10]$. Auch maligne Erkrankungen wie die chronisch lymphatische Leukämie, Polyzythämia vera und Bronchialkarzinome wurden beobachtet [11]. Für derartige Erkrankungen gab es bei unserer Patientin jedoch keinen Hinweis.

Das in der Literatur beschriebene häufige Auftreten von Rezidiven konnte bei unserer Patientin in einem Zeitraum von 4 Monaten nicht beobachtet werden.

Die bisher geringe Patientenzahl und die vielfältigen kasuistischen Therapieberichte lassen kaum Rückschlüsse über die Effektivität der verschiedenen Therapieoptionen zu, insbesondere da Spontanremissionen häufig beobachtet werden.

Die Gabe von systemischen Steroiden, zum Teil in Kombination mit Dapson und Antihistaminika wird dennoch empfohlen [12]. Bei therapierefraktären Fällen wurden Remissionen unter Cyclosporin gesehen [13]. Auch orale PUVA-Therapie und Minocyclin sollen einen günstigen Einfluss haben [14,15].

Die Therapie mit topischen Steroiden wurde bei unserer Patientin im akuten Stadium kurzzeitig nach Auftreten der Hautveränderungen begonnen. Der Juckreiz und die Hautveränderungen waren innerhalb von wenigen Tagen nach Therapiebeginn deutlich gebessert.

Daher halten wir auch einen Therapieversuch mit lokalen Steroiden in frühen Stadien der Erkrankung für gerechtfertigt. Die erfolgreiche Anwendung topischer Steroide wurde auch von Marks et al. 1980 und Moossavi et al. 2003 kasuistisch berichtet [16,17].
Zusammenfassend kann gesagt werden, dass das Wells-Syndrom eine seltene, wohl polyätiologische Erkrankung ist, die diagnostisch durch die Klinik und typische Histologie gesichert wird.

Die Erkrankung sollte differenzialdiagnostisch bei urtikariellen Hautveränderungen als seltene Ursache berücksichtigt werden. Nach Ausschluss systemischer Erkrankungen kommt als Therapie in erster Linie die Behandlung mit systemischen und/oder lokalen Kortikosteroiden zum Einsatz.

\section{Literatur}

${ }^{1}$ Wells GC. Recurrent granulomatous dermatitis with eosinophilia. Trans St Johns Hosp Dermatol Soc 1971; 57: 46-56

2 Wells GC, Smith NP. Eosinophilic cellulitis. Br J Dermatol 1979; 100: $101-109$

${ }^{3}$ Sommer S, Wilkinson SM, Merchant WJ. Eosinophilic cellulitis following the lines of Blaschko. Clin Exp Dermatol 1999; 24: 449-451

${ }^{4}$ Holme SA, McHenry P. Nodular presentation of eosinophilic cellulitis (Wells' syndrome). Clin Exp Dermatol 2000; 26: 677-679

${ }^{5}$ Mitchell AJ, Anderson TF, Headington JT, Rasmussen JE. Recurrent granulomatous dermatitis with eosinophilia. Wells' syndrome. Int J Dermatol 1984; 23: 198-202

${ }^{6}$ Dijkstra JW, Bergfeld WF, Steck WD, Tuthill RJ. Eosinophilic cellulitis associated with urticaria. A report of two cases. J Am Acad Dermatol 1986; 14: $32-38$

${ }^{7}$ Schorr WF, Tauscheck AL, Dickson KB, Melski JW. Eosinophilic cellulitis (Wells' syndrome): histologic and clinical features in arthropod bite reactions. J Am Acad Dermatol 1984; 11: 1043 - 1049

${ }^{8}$ Stern JB, Sobel HJ, Rotchford JP. Wells syndrome: is there collagen damage in the flame figures? Cuatn Pathol 1984; 11: 501 - 505

${ }^{9}$ Tsuji Y, Kawashima T, Yokota K, Tateishi Y, Kobayashi H, Itho A, Shimizu H. Wells syndrome as a manifestation of hypereosinophilic syndrome. Br J Dermatol 2002; 147: 811 - 812

${ }^{10}$ Schuttelaar ML, Jonkman MF. Bullous eosinophilic cellulitis (Wells syndrome) associated with Churg-Strauss syndrome. J Euro Acad Dermatol Venerol 2003; 17: 91 -93

${ }^{11}$ Farrar CW, Guerin DM, Wilson NJE. Eosinophilic cellulitis associated with squamous cell carcinoma of the bronchus. Br J Dermatol 2001; 145: $678-679$

${ }^{12}$ Lee MW, Nixon RL. Eosinophilic cellulitis case report: treatment options. Austral J Dermatol 1994; 35: 95-97

${ }^{13}$ Herr H, Koh JK. Eosinophilic cellulitis (Wells Syndrome) successfully treated with low-dose cyclosporine. J Korean Med Sci 2001; 16: $664-668$

${ }^{14}$ Stam-Westerveld EB, Daenen S, van der Meer JB, Jonkman MF. Eosinophilic cellulitis (Wells' syndrome): treatment with minocycline. Acta Derm Venereol 1998; 78: 157

${ }^{15}$ Diridl E, Hönigsmann $\mathrm{H}$, Tanew A. Wells syndrome responsive to PUVA therapy. Br J Dermatol 1997; 137: 479-480

${ }^{16}$ Marks R. Eosinophilic cellulitis - a response to treatment with dapsone: case report. Aust J Derm 1980; 21: 10-12

${ }^{17}$ Moossavi M, Mehregan DR. Wells' syndrome: a clinical and histopathologic review of seven cases. Int J Derm 2003; 42: 62 -67 